\title{
Swift/XRT observes the fifth outburst of the periodic supergiant fast X-ray transient IGR J11215-5952^
}

\author{
P. Romano ${ }^{1,2}$, L. Sidoli ${ }^{3}$, V. Mangano ${ }^{4}$, S. Mereghetti $^{3}$, and G. Cusumano ${ }^{4}$ \\ 1 INAF, Osservatorio Astronomico di Brera, via E. Bianchi 46, 23807 Merate (LC), Italy \\ e-mail: patrizia.romano@brera.inaf.it \\ 2 Università degli Studi di Milano, Bicocca, Piazza delle Scienze 3, 20126 Milano, Italy \\ 3 INAF, Istituto di Astrofisica Spaziale e Fisica Cosmica, via E. Bassini 15, 20133 Milano, Italy \\ ${ }^{4}$ INAF, Istituto di Astrofisica Spaziale e Fisica Cosmica, via U. La Malfa 153, 90146 Palermo, Italy
}

Received 1 March 2007 / Accepted 4 April 2007

\section{ABSTRACT}

\begin{abstract}
Context. The hard X-ray transient source IGR J11215-5952 was discovered in April 2005 with INTEGRAL and is a confirmed member of the new class of high mass X-ray binaries, the supergiant fast X-ray transients (SFXTs). Archival INTEGRAL data and RXTE observations have shown that the outbursts occur with a periodicity of $\sim 330$ days. Thus, IGR J11215-5952 is the first SFXT displaying periodic outbursts, possibly related to the orbital period.

Aims. We performed a target of opportunity observation with Swift with the main aim of monitoring the source behaviour around the time of the fifth outburst, expected on 2007 Feb. 9.

Methods. The source field was observed with Swift twice a day ( $2 \mathrm{ks} /$ day) starting from 2007 Feb. 4 until the fifth outburst, and then for $\sim 5 \mathrm{ks}$ a day afterwards, during a monitoring campaign that lasted 23 days for a total on-source exposure of $\sim 73 \mathrm{ks}$. This is the most complete monitoring campaign of an outburst from an SFXT.

Results. The spectrum during the brightest flares is described well by an absorbed power law with a photon index of 1 and $N_{\mathrm{H}} \sim$ $1 \times 10^{22} \mathrm{~cm}^{-2}$. A $1-10 \mathrm{keV}$ peak luminosity of $\sim 10^{36} \mathrm{erg} \mathrm{s}^{-1}$ was derived (assuming $6.2 \mathrm{kpc}$, the distance of the optical counterpart). Conclusions. These Swift observations are a unique data-set for an outburst of an SFXT, thanks to the combination of sensitivity and time coverage, and they allowed a study of IGR J11215-5952 from outburst onset to almost quiescence. We find that the accretion phase lasts longer than previously thought on the basis of lower-sensitivity instruments observing only the brightest flares. The observed phenomenology is consistent with a smoothly increasing flux triggered at the periastron passage in a wide eccentric orbit with many flares superimposed, possibly due to episodic or inhomogeneous accretion.
\end{abstract}

Key words. X-rays: stars - X-rays: individual: IGR J11215-5952

\section{Introduction}

The hard X-ray transient IGR J11215-5952 was discovered with the INTEGRAL satellite during an outburst in April 2005 (Lubinski et al. 2005) and was associated with HD 306414 (Negueruela et al. 2005), a B1Ia supergiant located at a distance of $6.2 \mathrm{kpc}$ (Masetti et al. 2006). The short duration of the outburst, together with the likely optical counterpart, suggested that IGR J11215-5952 could be a new member of the class of supergiant fast X-ray transients (SFXTs; Negueruela et al. 2006). Analysing archival INTEGRAL observations of the source field, Sidoli et al. (2006, hereafter Paper I) discovered two previously unnoticed outbursts (in July 2003 and in May 2004) that demonstrate the recurrent nature of this transient and suggest a possible periodicity of $\sim 330$ days. This periodicity was confirmed by the detection of the fourth outburst from IGR J11215-5952 with RossiXTE/PCA on 2006 March 16-17, 329 days after the third outburst (Smith et al. 2006b). The RXTE/PCA observations showed strong flux variability and a hard spectrum (powerlaw photon index of $1.7 \pm 0.2$ in the range $2.5-15 \mathrm{keV}$ ), as well as

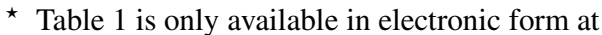
http://www . aanda. org a possible pulse period of $\sim 195 \mathrm{~s}$ (Smith et al. 2006a). The periodicity was confirmed with RXTE observations of the latest outburst, yielding $P=186.78 \pm 0.3 \mathrm{~s}$ (Swank et al. 2007). Followup observations with Swift/XRT refined the source position and confirmed the association with HD 306414 (Steeghs et al. 2006). A hard power-law with a high-energy cut-off around $15 \mathrm{keV}$ is a good fit to the spectra observed with INTEGRAL (Paper I). For the distance of $6.2 \mathrm{kpc}$, the peak fluxes of the outbursts correspond to a luminosity of $\sim 3 \times 10^{36} \mathrm{erg} \mathrm{s}^{-1}(5-100 \mathrm{keV})$. All these findings confirmed IGR J11215-5952 as a member of the class of the SFXT, and as the first object of this class of high mass X-ray binaries displaying periodic outbursts.

Predicting a fifth outburst for 2007 Feb. 9, we obtained a Target of Opportunity (ToO) observing campaign with Swift, which commenced on Feb. 4. The source started showing renewed activity on Feb. 8 (Romano et al. 2007) and underwent a powerful outburst on Feb. 9 (Mangano et al. 2007a,b; Sidoli et al. 2007; Swank et al. 2007). This paper presents our observations of IGR J11215-5952, and it is organised as follows. In Sect. 2 we describe our observations and data reduction; in Sect. 3 we describe our spatial, timing, and spectral data analysis. Finally, in Sect. 4 we discuss our findings and draw our conclusions. 

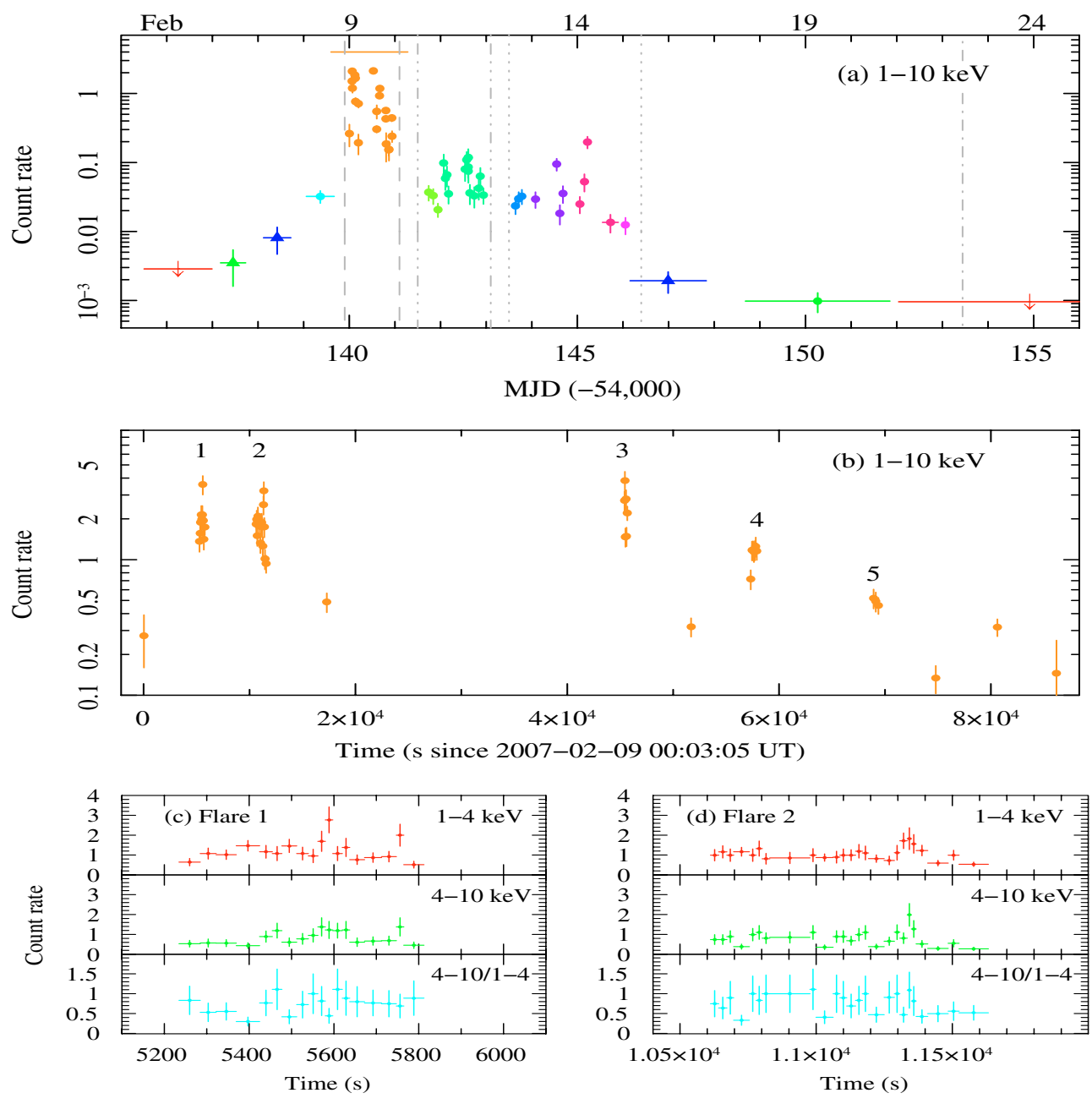

Fig. 1. XRT light curves, corrected for pile-up, PSF losses, and vignetting and background-subtracted. a) The $1-10 \mathrm{keV}$ light curve for the whole campaign. Different colours denote different observations (Table 1), and points before Feb. 6 (MJD 54 137) and after Feb. 15 (MJD 54 146) are drawn from the sum of several observations. Filled circles are full detections $(S / N>3)$, triangles marginal detections $(2<S / N<3)$, while downward-pointing arrows are 3- $\sigma$ upper limits. The vertical lines mark our time selections for spectroscopic analysis (observation 6, observations 7-8, observations 9-12, end of observation 18). The horizontal line marks the region shown enlarged in panel b). b) Detail of observation 6, with a binning that allowed us to achieve an $S / N$ in excess of 6 . The numbers mark the 5 flares on which we performed spectroscopic analysis. c) Detail of flare 1, showing the $1-4 \mathrm{keV}, 4-10 \mathrm{keV}$ count rates (top and middle panel) and the hardness ratio 4-10/1-4 (bottom). The data were rebinned in order to have at least 20 counts per bin in both the 1-4 keV and 4-10 keV bands. d) Same as c) for flare 2 .

\section{Observations and data reduction}

Table 1 reports the $\log$ of the Swift/XRT observations used for this work. Thanks to Swift's fast-slewing and flexible observing scheduling, the ToO observations started on 2007 Feb. 4 with $2 \mathrm{ks}$ per day evenly spread throughout the day to maximise the chances of detection of the outburst onset, and were increased to $5 \mathrm{ks}$ afterwards for a total of 23 days and a total on-source exposure of $\sim 73 \mathrm{ks}$. From the Swift Archive we also retrieved the data from a $643 \mathrm{~s}$ ToO performed on 2006 Mar. 20 during the fourth outburst of this source (Steeghs et al. 2006).

The XRT data were processed with standard procedures (xrtpipeline v0.10.6), filtering, and screening criteria by using FTOOLS in the Heasoft package (v.6.1.2). Given the low rate of the source during the whole campaign, we only considered photon-counting data (PC) and further selected XRT grades 0-12 (Burrows et al. 2005). With the exception of observation 6 (2006 Feb. 9), the data show an average count rate of $<0.5$ counts $^{-1}$ and no pile-up correction was necessary. We therefore extracted the source events from a circular region with a radius of 11 pixels ( 1 pixel $\left.\sim 2.37^{\prime \prime}\right)$. During observation 6, pile-up correction was required and we adopted an annular source extraction region with radii 4 and 30 pixels. To account for the background, we also extracted events within an annular region centred on the source and with radii 40 and 100 pixels, free from background sources. Ancillary response files were generated with xrtmkarf, and they account for different extraction regions, vignetting, and PSF corrections. We used the latest spectral redistribution matrices (v008) in the Calibration Database maintained by HEASARC. For timing analysis, the arrival times of XRT events were converted to the Solar System barycentre with the task barycorr and source events were extracted from the circular region with 30 pixels radius to maximise statistics.

BAT always observed IGR J11215-5952 simultaneously with XRT, but only survey data products, in the form of detector plane histograms (DPH) with typical integration time of $\sim 300 \mathrm{~s}$, are available. The BAT data were analysed using the standard BAT analysis software distributed within FTOOLS. The DPH data were calibrated with the task baterebin using the proper BAT gain/offset files from the housekeeping data directory, and sky images from each observation were extracted in the $15-25,25-50,50-100,100-150$, and $15-150 \mathrm{keV}$ energy bands. The batcelldetect task never detected the source above a signal-to-noise ratio $(S / N)$ threshold of 4 . This is consistent with the extrapolation at the high energies of the XRT data fit (Sect. 3) with an absorbed power-law with exponential cutoff, with e-folding energy of $15 \pm 2 \mathrm{keV}$ drawn from the RXTE fit (Swank et al. 2007).

Throughout this paper the uncertainties are given at a $90 \%$ confidence level for one interesting parameter (i.e., $\Delta \chi^{2}=$ 2.71), unless otherwise stated. The spectral indices are parameterized as $F_{v} \propto v^{-\alpha}$, where $F_{v}\left(\mathrm{erg} \mathrm{cm}^{-2} \mathrm{~s}^{-1} \mathrm{~Hz}^{-1}\right)$ is the flux density as a function of frequency $v$; we also use $\Gamma=\alpha+1$ as the photon index, $N(E) \propto E^{-\Gamma}\left(\mathrm{ph} \mathrm{cm}^{-2} \mathrm{~s}^{-1} \mathrm{keV}^{-1}\right)$. 


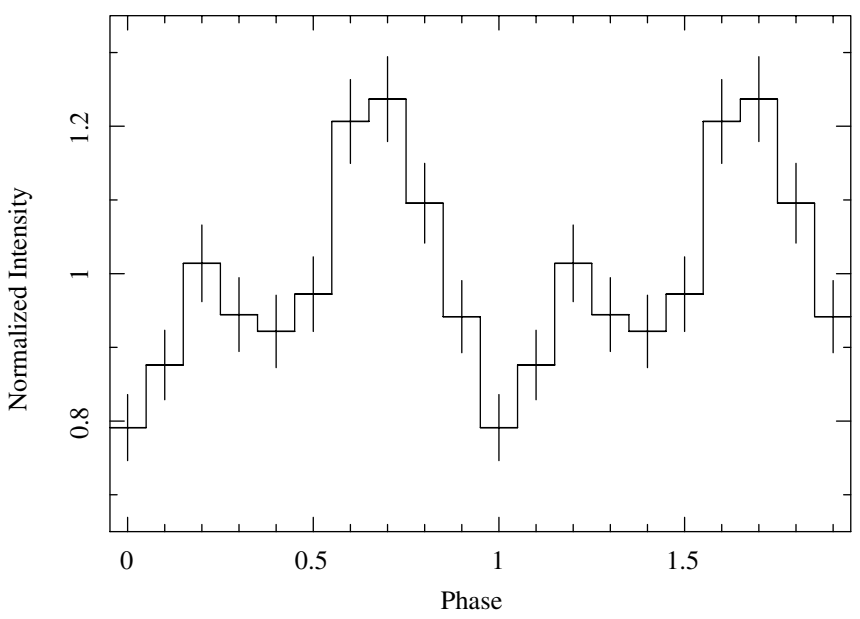

Fig. 2. Folded $0.2-10 \mathrm{keV}$ light curve of the combined observations 6 though 11, using a period of $186.78 \mathrm{~s}$ (Swank et al. 2007).

\section{Analysis and results}

A refined position was obtained by summing all data taken in 2007 with the exclusion of observation 6 (affected by pileup) at $\mathrm{RA}(\mathrm{J} 2000)=11^{\mathrm{h}} 21^{\mathrm{m}} 46^{\mathrm{s}} .90, \operatorname{Dec}(\mathrm{J} 2000)=-59^{\circ} 51^{\prime} 46^{\prime \prime} \cdot 9$, with an error, drawn from the cross-correlation with the USNOB1.0 catalogue, of 1". 1 (90\% confidence). This position is 1".2 from the optical counterpart HD 306414.

We extracted light curves in the $1-10 \mathrm{keV}$ (total), $1-4 \mathrm{keV}$ (soft), and 4-10 keV (hard) bands. The $0.2-1 \mathrm{keV}$ band was not used in our analysis because, given the high absorbing column density, its signal was significantly lower than the one of the other bands. The light curves were corrected for pointspread function (PSF) losses, due to the extraction region geometry, bad/hot pixels, and columns falling within this region, and for vignetting by using the task xrtlccorr (v0.1.9), which generates an orbit-by-orbit correction based on the instrument map. We then subtracted the scaled background rate in each band from their respective source light curves and calculated the 4-10/1-4 hardness ratio. The IGR J11215-5952 light curve (Fig. 1) shows an increase in count rate by a factor of $\sim 10$ in less than $1.5 \mathrm{~h}$ and of a factor of $\sim 65$ in $17 \mathrm{~h}$ on 2007 Feb. 9. However, no significant variation in the hardness ratio can be proven (panels c,d of Fig. 1). Indeed, fitting the hardness ratio as a function of time (or as a function of count rate) to a constant model yields a value of $0.49 \pm 0.03$ and $\chi^{2}=1.13$ for 80 degrees of freedom (d.o.f.). We folded the data at the period of $186.78 \mathrm{~s}$ reported by Swank et al. (2007) based on the Feb. 9 01:20-03:20 UT RXTE/PCA observations and obtained the $0.2-10 \mathrm{keV}$ light curve shown in Fig. 2.

Upon examination of the light curve presented in Fig. 1 and the available counting statistics, we selected different time bins over which we accumulated spectra. These include $i$ ) the quiescent phase before the 2007 Feb. 9 outburst, ii) the Feb. 9 outburst (observation 6), and iii) the tail phase of the outburst (observations 7-8, 9-12,7-12,7-18). We then selected 5 flaring episodes from observation 6 (see Fig. 1b). A comparison with the data collected during the tail of the 2006 outburst was also performed. The data were rebinned with a minimum of 20 counts per energy bin to allow $\chi^{2}$ fitting. However, the Cash statistic (Cash 1979) and spectrally unbinned data were used for the 2006 observation ( 28 counts), before the onset of the outburst (41 counts), for the late flares, and the late observations. The spectra were all fit with XSPEC (v11.3.2) in the $0.5-9 \mathrm{keV}$
Table 1. Spectral fit results.

\begin{tabular}{llrrr}
\hline \hline Spectrum $^{a}$ & $\begin{array}{l}N_{\mathrm{H}} \\
\left(10^{22} \mathrm{~cm}^{-2}\right)\end{array}$ & $\Gamma$ & $\begin{array}{r}\chi^{2}(\text { d.o.f.) } \\
\text { C-stat }(\%)^{c}\end{array}$ & $\begin{array}{r}L_{1-10 \mathrm{kev}}^{b} \\
\left(\mathrm{erg} \mathrm{s}^{-1}\right)\end{array}$ \\
\hline 001 (2006) & $0.88_{-0.62}^{+0.96}$ & $1.89_{-0.92}^{+1.07}$ & $167.7(65.8 \%)$ & $2.35 \times 10^{-1}$ \\
001-005 & $2.28_{-1.50}^{+2.21}$ & $1.34_{-0.96}^{+1.11}$ & $225.9(65.8 \%)$ & $4.32 \times 10^{-2}$ \\
006 & $1.04_{-0.20}^{+0.25}$ & $1.00_{-0.14}^{+0.16}$ & $1.04(83)$ & 4.78 \\
006 Flare 1 & $0.85_{-0.32}^{+0.46}$ & $0.94_{-0.28}^{+0.31}$ & $0.66(17)$ & 8.68 \\
006 Flare 2 & $1.11_{-0.49}^{+0.79}$ & $0.91_{-0.32}^{+0.42}$ & $1.16(25)$ & 8.32 \\
006 Flare 3 & $0.83_{-0.42}^{+0.62}$ & $0.82_{-0.40}^{+0.44}$ & $0.93(10)$ & 11.1 \\
006 Flare 4 & $0.88_{-0.31}^{+0.38}$ & $1.03_{-0.31}^{+0.32}$ & $623.6(39.0 \%)$ & 4.86 \\
006 Flare 5 & $2.02_{-0.79}^{+1.01}$ & $1.51_{-0.53}^{+0.58}$ & $436.0(54.6 \%)$ & 2.75 \\
007-008 & $1.75_{-0.83}^{+1.33}$ & $1.94_{-0.60}^{+0.64}$ & $518.3(66.0 \%)$ & $2.22 \times 10^{-1}$ \\
009-012 & $1.04_{-0.36}^{+0.47}$ & $1.48_{-0.35}^{+0.39}$ & $576.9(53.6 \%)$ & $8.82 \times 10^{-2}$ \\
007-012 & $1.86_{-0.44}^{+0.68}$ & $1.92_{-0.38}^{+0.43}$ & $1.09(18)$ & $1.40 \times 10^{-1}$ \\
007-018 & $2.04_{-0.50}^{+0.62}$ & $2.08_{-0.37}^{+0.41}$ & $1.19(19)$ & $5.96 \times 10^{-2}$ \\
\hline
\end{tabular}

${ }^{a}$ Last three digits of observation numbers, see Table 1, Col. 1.

${ }^{b}$ Luminosity in the $1-10 \mathrm{keV}$ band in units of $10^{35} \mathrm{erg} \mathrm{s}^{-1}$ obtained from the spectral fits.

${ }^{c}$ Cash statistics (C-stat) and percentage of Monte Carlo realizations that had statistic $<$ C-stat. We performed $10^{4}$ simulations.

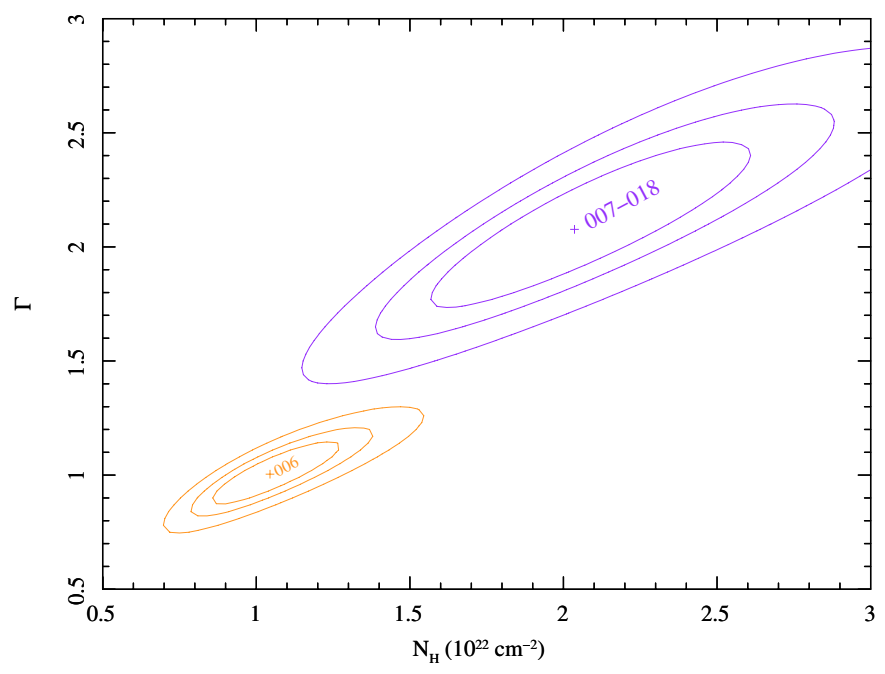

Fig. 3. XRT time-selected spectroscopy. The $\Delta \chi^{2}=2.3,4.61,9.21$ contour levels for the column density in units of $10^{22} \mathrm{~cm}^{-2}$ vs. the photon index, with best-fit values indicated by crosses. Orange contours are from observation 6 , while the blue ones are from the combined observations 7 though 18 .

energy range, adopting the typical pulsar spectral model, an absorbed power law model.

The best-fit parameters are reported in Table 1 along with the mean luminosity of each time selection. The spectrum of the brightest part of the outburst (observation 6) could be fit with a single power law, with a photon index $\Gamma=1.00_{-0.14}^{+0.16}$, and with an absorbing column density of $N_{\mathrm{H}}=\left(1.04_{-0.20}^{+0.25}\right) \times$ $10^{22} \mathrm{~cm}^{-2}\left(\chi_{\text {red }}^{2}=1.04 / 83\right.$ d.o.f. $)$, while the combined observations $7-18$ yielded a photon index of $2.08_{-0.37}^{+0.41}$ and $N_{\mathrm{H}}=$ $\left(2.04_{-0.50}^{+0.62}\right) \times 10^{22} \mathrm{~cm}^{-2}\left(\chi_{\text {red }}^{2}=1.19 / 19\right.$ d.o.f. $)$. We note that the $1-10 \mathrm{keV}$ count rate to unabsorbed $1-10 \mathrm{keV}$ flux conversion factor, obtained from the best-fit model for observation 6 , is $2.9 \times 10^{-10} \mathrm{erg} \mathrm{cm}^{-2}$ count $^{-1}$. We also performed fits of observation 6 with an absorbed black-body. Since the column density assumed a value significantly below that resulting from the interstellar medium, the power law fit was favoured. In all cases the best-fit absorbing column density is consistent 
(within 2- $\sigma$ ) with the Galactic absorption along the line of sight of IGR J11215-5952. This value is significantly lower than the column density measured with RXTE/PCA during the 2006 outburst (Smith et al. 2006b), $(11 \pm 3) \times 10^{22} \mathrm{~cm}^{-2}$, which is likely overestimated because it was derived with RXTE/PCA in the energy range $2.5-15 \mathrm{keV}$.

To investigate spectral variations, we created the contour levels for the column density vs. the photon index. The most interesting example is shown in Fig. 3. They indicate that the photon index showed significant variations, with the spectrum softening as the outburst progresses, confirming the 2006 observation of the outburst tail; there is also evidence of an increasing absorbing column density. These findings are independent of our choice of an absorbed power-law model.

\section{Discussion and conclusions}

We have carried out the most complete monitoring campaign of an outburst from an SFXT, thanks to the known periodicity of the outburst activity from IGR J11215-5952 (Paper I). This is remarkable, since the transient and unpredictable nature of the outbursts from all other SFXTs hampers a similarly extensive study from the almost "quiescent" level up to the "flaring" activity. The entire "outburst event" was monitored for 23 days. The source was under the threshold of detectability in the early days of the campaign, with a luminosity below $3.7 \times 10^{33} \mathrm{erg} \mathrm{s}^{-1}$. On Feb. 9 the source underwent a bright outburst up to $\sim 1.1 \times$ $10^{36} \mathrm{erg} \mathrm{s}^{-1}$. The bright part of the outburst (Feb. 9; see Fig. 1b) is composed of at least five flares with variable peak flux, each with a duration of $\sim 15 \mathrm{~min}-2 \mathrm{~h}$. This bright flaring activity lasted about 1 day, then the source underwent a decline phase that was not flat, but composed of other equally short flares, one order of magnitude fainter. This decline phase lasted about 5 days, and then the source faded to a much fainter level, almost below the threshold of detectability. The whole outburst lasted about $15 \mathrm{~d}$, after which the source became fainter than $1.2 \times 10^{33} \mathrm{erg} \mathrm{s}^{-1}$. Thus, IGR J11215-5952 reached the typical luminosity of SFXTs during outburst (around $10^{36} \mathrm{erg} \mathrm{s}^{-1}$ ), showing a dynamic range larger than $10^{3}$ and a hard X-ray spectrum proper to this kind of sources. The brightest part of the outburst lasted less than a day, on Feb. 9, and would have been the only flaring activity seen with less sensitive instruments. Indeed up to now, observations of outbursts from SFXTs have mostly been performed with instruments on-board RXTE and/or INTEGRAL, which could only catch the brightest flares and which missed the complete evolution of the phenomenon, from the onset of the outburst, and down again to the level of almost quiescence, which is expected at a level around $\sim 10^{32} \mathrm{erg} \mathrm{s}^{-1}$ (possible magnetospheric emission plus the contribution from the soft X-ray emission from the OB supergiant). Only during XMM-Newton and Chandra observations of IGR J17544-2619 (González-Riestra et al. 2004; in't Zand 2005) were outbursts observed starting from the quiescent emission, but the "postflare" phases could not be followed completely and thus the duration of the entire outburst phase could not be measured.

For IGR J11215-5952 we could exceptionally observe the whole phenomenon, which for the first time reveals that the "short outbursts" (the "flares" lasting minutes or few hours) are actually part of a much longer "outburst event" (lasting several days), which we believe is triggered at the periastron passage in a wide, highly eccentric orbit. Indeed, the IGR J11215-5952 outburst recurrence time (329 days) is remarkably stable and reveals an underlying clock, which can be naturally associated with the orbital motion in a non-circular orbit. The most prominent short flares on Feb. 9 are probably produced either by the episodic accretion of clumps from the massive wind (Owocki \& Cohen 2006) or by an inhomogeneous accretion stream near periastron (similar to what has been proposed to explain the periodic outbursts from the eccentric X-ray pulsar GX 302-1, e.g. Leahy 1991). Thus, both mechanisms originally proposed to explain the SFXTs outbursts, seem to be at work in IGR J11215-5952, i.e., accretion at periastron passage in a wide eccentric orbit (Negueruela et al. 2006) and accretion from clumpy winds, (in't Zand 2005). Applying a spherically symmetric homogeneous wind model to a B1 Ia spectral type companion with a mass of $39 M_{\odot}, 42$ solar radii, and a wind mass loss of $3.67 \times 10^{-6} M_{\odot} \mathrm{yr}^{-1}$ (Vink et al. 2000), the short outburst duration implies an eccentricity higher than 0.9 .

From the spectroscopy of the single flares there is evidence for only minor variations in the local absorbing column density (which would suggest the clear presence of clumps). This may be partly due to the high column density along the line of sight that absorbs most of the radiation below $1 \mathrm{keV}$, thus preventing us from detecting comparatively small variations in the intrinsic column density. However, XRT data show evidence of softening of the spectrum in the long decay to a quiescent state (thus confirming the 2006 observation of the outburst tail) and possible evidence of an $N_{\mathrm{H}}$ growth connected with the same transition.

Acknowledgements. We thank the Swift team for making these observations possible, in particular the duty scientists and science planners M. Chester, S. Hunsberger, J. Kennea, C. Pagani, and J. Racusin; we thank N. Gehrels for approving this ToO and D. Burrows for a winning observing strategy. We thank S. Campana, P. D’Avanzo, A. Paizis, P. Persi, V. F. Polcaro, and S. Vercellone for insightful discussions. This research has made use of NASA's Astrophysics Data System Bibliographic Services, as well as the NASA/IPAC Extragalactic Database (NED), which is operated by the Jet Propulsion Laboratory, California Institute of Technology, under contract with the National Aeronautics and Space Administration. This work was supported by MIUR grant 2005-025417 and contract ASI/INAF I/023/05/0. P.R. thanks INAF-IASFMi, where most of the work was carried out, for their kind hospitality.

\section{References}

Burrows, D. N., Hill, J. E., Nousek, J. A., et al. 2005, Space Sci. Rev., 120, 165 Cash, W. 1979, ApJ, 228, 939

González-Riestra, R., Oosterbroek, T., Kuulkers, E., Orr, A., \& Parmar, A. N. 2004, A\&A, 420, 589

in't Zand, J. J. M. 2005, A\&A, 441, L1

Leahy, D. A. 1991, MNRAS, 250, 310

Lubinski, P., Bel, M. G., von Kienlin, A., et al. 2005, ATel, 469

Mangano, V., Romano, P., \& Sidoli, L. 2007a, ATel, 995

Mangano, V., Romano, P., \& Sidoli, L. 2007b, ATel, 996

Masetti, N., Pretorius, M. L., Palazzi, E., et al. 2006, A\&A, 449, 1139

Negueruela, I., Smith, D. M., \& Chaty, S. 2005, ATel, 470

Negueruela, I., Smith, D. M., Reig, P., Chaty, S., \& Torrejón, J. M. 2006, in Proceedings of the The X-ray Universe 2005, 26-30 September 2005, El Escorial, Madrid, Spain, ed. A. Wilson, ESA SP-604, 165

Owocki, S. P., \& Cohen, D. H. 2006, ApJ, 648, 565

Romano, P., Sidoli, L., \& Mangano, V. 2007, ATel, 994

Sidoli, L., Paizis, A., \& Mereghetti, S. 2006, A\&A, 450, L9

Sidoli, L., Mereghetti, S., Vercellone, S., et al. 2007, ATel, 997

Smith, D. M., Bezayiff, N., \& Negueruela, I. 2006a, ATel, 773

Smith, D. M., Bezayiff, N., \& Negueruela, I. 2006b, ATel, 766

Steeghs, D., Torres, M. A. P., \& Jonker, P. G. 2006, ATel, 768

Swank, J., Smith, D., \& Markwardt, C. 2007, ATel, 997

Vink, J. S., de Koter, A., \& Lamers, H. J. G. L. M. 2000, A\&A, 362, 295 
P. Romano et al.: The fifth outburst of IGR J11215-5952 observed by Swift, Online Material p 1

\section{Online Material}


P. Romano et al.: The fifth outburst of IGR J11215-5952 observed by Swift, Online Material p 2

Table 1. Observation log.

\begin{tabular}{lllll}
\hline \hline Sequence & Start time (MJD) & $\begin{array}{l}\text { Start time (UT) } \\
\text { (yyyy-mm-dd hh:mm:ss) }\end{array}$ & $\begin{array}{l}\text { End time (UT) } \\
\text { (yyyy-mm-dd hh:mm:ss) }\end{array}$ & $\begin{array}{l}\text { Net Exposure }^{a} \\
\text { (s) }\end{array}$ \\
\hline 00030384001 & 53814.7853 & $2006-03-2018: 50: 47$ & $2006-03-2019: 01: 53$ & 643 \\
00030881001 & 54135.5060 & $2007-02-0412: 08: 34$ & $2007-02-0423: 38: 58$ & 2048 \\
00030881002 & 54136.5092 & $2007-02-0512: 13: 12$ & $2007-02-05$ 23:44:57 & 1865 \\
00030881003 & 54137.1798 & $2007-02-0604: 18: 55$ & $2007-02-0617: 28: 17$ & 1941 \\
00030881004 & 54138.1244 & $2007-02-0702: 59: 06$ & $2007-02-0717: 13: 56$ & 1213 \\
00030881005 & 54139.0604 & $2007-02-0801: 27: 02$ & $2007-02-0816: 03: 57$ & 1403 \\
00030881006 & 54140.0021 & $2007-02-0900: 03: 05$ & $2007-02-0923: 59: 57$ & 4668 \\
00030881007 & 54141.6747 & $2007-02-1016: 11: 33$ & $2007-02-1100: 16: 56$ & 3141 \\
00030881008 & 54142.0696 & $2007-02-1101: 40: 15$ & $2007-02-1200: 10: 11$ & 4232 \\
00030881009 & 54143.6078 & $2007-02-1214: 35: 17$ & $2007-02-1219: 38: 57$ & 3337 \\
00030881010 & 54144.0085 & $2007-02-1300: 12: 17$ & $2007-02-1316: 30: 58$ & 3091 \\
00030881011 & 54145.0182 & $2007-02-1400: 26: 09$ & $2007-02-1421: 25: 56$ & 4521 \\
00030881012 & 54146.0142 & $2007-02-1500: 20: 26$ & $2007-02-1509: 58: 57$ & 4590 \\
00030881013 & 54147.6084 & $2007-02-1614: 36: 05$ & $2007-02-1619: 44: 56$ & 5230 \\
00030881014 & 54148.6848 & $2007-02-1716: 26: 09$ & $2007-02-1721: 23: 57$ & 4295 \\
00030881015 & 54149.2812 & $2007-02-1806: 44: 59$ & $2007-02-1812: 01: 58$ & 4804 \\
00030881016 & 54150.2187 & $2007-02-1905: 14: 52$ & $2007-02-1912: 02: 56$ & 4636 \\
00030881017 & 54151.6337 & $2007-02-2015: 12: 28$ & $2007-02-2020: 19: 56$ & 4847 \\
00030881018 & 54152.0412 & $2007-02-2100: 59: 16$ & $2007-02-2117: 11: 57$ & 5194 \\
00030881019 & 54153.4431 & $2007-02-2210: 38: 04$ & $2007-02-2215: 39: 58$ & 3814 \\
00030881021 & 54155.1683 & $2007-02-2404: 02: 20$ & $2007-02-2413: 46: 57$ & 2963 \\
00030881023 & 54157.5108 & $2007-02-2612: 15: 37$ & $2007-02-2618: 48: 58$ & 786 \\
\hline
\end{tabular}

${ }^{a}$ The exposure time is spread over several snapshots (single continuous pointings at the target) during each observation. 